CERN-TH.6762/92

\title{
On amplitude zeros at threshold
}

\author{
E.N. Argyres* and Costas G. Papadopoulos \\ TH Division, CERN, Geneva, Switzerland \\ Ronald H.P. Kleiss, \\ NIKHEF-H, Amsterdam, the Netherlands
}

\begin{abstract}
The occurrence of zeros of $\mathcal{A}(2 \rightarrow n)$ amplitudes at threshold in scalar theories is studied. We find a differential equation for the scalar potential, which incorporates all known cases where the $\mathcal{A}(2 \rightarrow n)$ amplitudes at threshold vanish for all sufficiently large $n$, in all space-time dimensions, $d \geq 1$. This equation is related to the reflectionless potentials of Quantum Mechanics and to integrable theories in 1+1 dimensions. As an application, we find that the sine-Gordon potential and its hyperbolic version, the sinh-Gordon potential, also have amplitude zeros at threshold, $\mathcal{A}(2 \rightarrow n)=0$, for $n \geq 4$ and $d \geq 2$, independently of the mass and the coupling constant.
\end{abstract}

CERN-TH.6762/92

December 1992

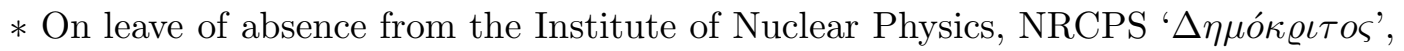
GR-153 10 Athens, Greece. 
The behaviour of amplitudes for processes with a large number of particles in the final state, originally connected to the problem of baryon-number violating processes at very high energies, has recently been studied in the context of normal perturbation theory as well [1, 2, 3]. Several properties of scalar tree-order amplitudes have been established:

1. The factorial growth of the amplitude of one virtual Higgs to go to $n$ onshell Higsses, $\mathcal{A}(1 \rightarrow n)$, at threshold has been found to be a generic property of monomial scalar theories, like $\frac{\lambda}{m !} \phi^{m}$, for $m \geq 3$ [2, 3], as well as of the spontaneously broken $\phi^{4}$ theory.

2. A lower bound on the tree-order cross section $\sigma\left(f \bar{f} \rightarrow H^{*} \rightarrow n H\right)$ can be established rigorously, yielding a unitarity-violating behaviour 因, 可.

The construction of a theory in which these amplitudes do not grow factorially with $n$ has been performed in [6]. In such a theory the potential differs from the free-field one only by factors of $\log \phi$, indicating that the unitarity-violation problem emerges when the anharmonic terms of the potential dominate over the harmonic one (as they will in any finite polynomial potential). The factorial growth is related to the radius of convergence of the generating function of the amplitudes [6], or equivalently to the pole structure of the classical space-independent field configurations [7] in the complex-time plane.

Trying to go beyond tree order, Voloshin [8, 9] discovered the phenomenon of nullification: for all $n$ larger than some $n_{0}$, the $\mathcal{A}(2 \rightarrow n)$ amplitudes vanish when the final-state scalars are produced at rest. In $\phi^{4}$ theory, $n_{0}$ was found to be 4 . The explicit form of the amplitudes $\mathcal{A}(2 \rightarrow n)$ for any $\phi^{m}$ scalar theory was given in ref.[10], where the nullification of $\mathcal{A}(2 \rightarrow n)$ amplitudes was found also for the $\phi^{3}$, with $n_{0}=3$, and for the broken symmetry $\phi^{4}$ theory, with $n_{0}=2$ (see also ref. [11]). This property is independent of the self-coupling or the mass of the scalar particle or the number of space-time dimensions (provided that $d \geq 2$ ); it is related only to the form of the potential. Nevertheless, it is not a trivial effect, as for instance the nullification of all $\mathcal{A}(2 \rightarrow 2 k+1)$ tree-order amplitudes in a $\phi^{2 n}$ theory, $n, k$ being integers, which holds for all kinematical configurations. Amplitude zeros emerge also in the cases when the $\phi$ field is coupled to other boson and/or fermion fields [9, [12]. As we will see below, this nullification requires definite relations between the masses and the couplings appearing in the Lagrangian.

In all known cases, nullification occurs when the form of the second derivative of the potential evaluated at the classical background corresponds to the well-known reflectionless potentials of Quantum Mechanics [13. In this note, we establish a differential equation involving only the potential and its derivatives with respect to the field, which, if satisfied, leads to nullification.

Let us assume a general scalar-field potential whose expansion (apart from the mass term and setting $m_{H}=1$ ) is given by

$$
V(\phi)=\sum_{m=3}^{\infty} \frac{\lambda_{m}}{m !} \phi^{m} .
$$




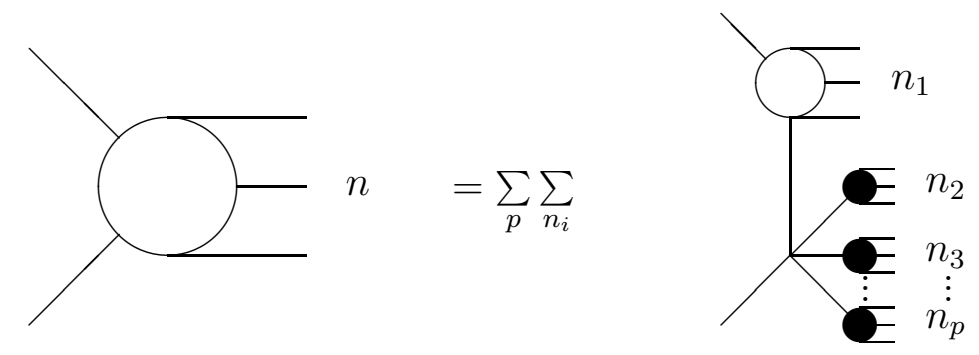

Figure 1: Diagrammatic representation of the recursion formula of Eq.(2). The black circles correspond to the $\mathcal{A}(1 \rightarrow n)$ amplitudes at threshold.

The $a_{2}(n) \equiv \mathcal{A}\left(H\left(p_{1}\right)+H\left(p_{2}\right) \rightarrow n H(q)\right)$ amplitudes are given by the following recursion formula (see Fig.凹):

$$
a_{2}(n)=-i \sum_{p=2}^{n} \frac{\lambda_{p+1}}{(p-1) !} \sum_{\substack{n_{2}, \ldots, n_{p} \geq 1 \\ n_{1}+\ldots+n_{p}=n}} \frac{i a_{2}\left(n_{1}\right)}{P\left(n_{1}\right)} \frac{i a\left(n_{2}\right)}{\left(n_{2}^{2}-1\right)} \cdots \frac{i a\left(n_{p}\right)}{\left(n_{p}^{2}-1\right)} \frac{n !}{n_{1} ! n_{2} ! \ldots n_{p} !}
$$

where $q^{\mu}=(1, \overrightarrow{0}), p_{1}^{\mu}=(E ; \vec{p}), p_{1}^{2}=1$ (recall that $\left.m_{H}=1\right), a(n) \equiv \mathcal{A}(1 \rightarrow n)$ is the amplitude at threshold and $P(n)$ is the inverse propagator, given by

$$
P(n)=\left(p_{1}-n q\right)^{2}-1=n(n-2 E)
$$

The ansätze

$$
a(n)=-i n !\left(n^{2}-1\right) b(n) \quad, \quad a_{2}(n)=-i n ! P(n) b_{2}(n),
$$

and the introduction of the generating functions

$$
f(x)=\sum_{n \geq 1} b(n) x^{n} \quad, \quad f_{2}(x)=\sum_{n \geq 0} b_{2}(n) x^{n+1}
$$

transform Eq.(2) into the following differential equation for $f_{2}(x)$ :

$$
x^{2} f_{2}^{\prime \prime}(x)-(1+2 E) x f_{2}^{\prime}(x)-\left[-1-2 E+V^{\prime \prime}(f(x))\right] f_{2}(x)=0,
$$

with initial conditions $f_{2}(0)=0$ and $f_{2}^{\prime}(0)=1$. The corresponding equation for $f(x)$ is 3

$$
x^{2} f^{\prime \prime}(x)+x f^{\prime}(x)=f(x)+V^{\prime}(f(x)),
$$

with initial conditions $f(0)=0$ and $f^{\prime}(0)=1$. Equation (77) can be viewed as the classical equation [14] for the field $\phi(\tau)=f(x)$ with the assignment $x=c e^{\tau}, \tau$ being the imaginary time. It can also be interpreted as the static soliton-like configuration in $1+1$ dimensions, $(t, z)$, where $x=c e^{z}$ (the constant $c$ guarantees the appropriate initial conditions for the $f(x)$, at $x=0, z=-\infty)$. 
Let us now assume that $V^{\prime \prime}(f(x))$ can be written as

$$
V^{\prime \prime}=-\frac{R}{\cosh ^{2}(A \tau)},
$$

where

$$
\tau=\int_{\phi(0)}^{\phi(\tau)} \frac{d \phi}{\sqrt{\phi^{2}+2 V(\phi)}}
$$

Then, Eq.(6) takes the form

$$
\left(\frac{d^{2}}{d \tau^{2}}-E^{2}+\frac{R}{\cosh ^{2}(A \tau)}\right) \psi(\tau)=0
$$

where $x=c e^{\tau}$ and $f_{2}(x)=e^{(E+1) \tau} \psi(\tau)$.

This is just the quantum-mechanical problem of a reflectionless potential: we must have an integer value for $s$, where $s(s+1) \equiv R A^{-2}$. The solution, regular at $\tau \rightarrow-\infty$, is given by 13

$$
\psi(\tau)=C\left(1-\xi^{2}\right)^{\frac{\epsilon}{2}} F\left(\epsilon-s, \epsilon+s+1 ; \epsilon+1 ; \frac{1}{2}(1+\xi)\right)
$$

where $C$ is determined by the initial conditions on $f_{2}(x), \epsilon=-E / A, \xi=\tanh (A \tau)$ and $F(a, b ; c ; z)$ is the hypergeometric function. The number of the poles or bound states, leading to non-zero amplitudes, is exactly equal to $s$ and the location of the poles is given by $E(k)=A k, k=1, \ldots, s$. This means that the only non-zero amplitudes occur when

$$
n=2 A k, \quad k=1, \ldots, s, \quad \mathcal{A}(2 \rightarrow n) \neq 0
$$

It is possible to eliminate the $\tau$ dependence from Eqs.(8) and (9) and obtain an equation wich involves only the potential and its derivatives:

$$
\left(\frac{U^{\prime \prime \prime}(\phi)}{2 A\left(1-U^{\prime \prime}(\phi)\right)}\right)^{2}=\frac{1-\left(1-U^{\prime \prime}(\phi)\right) R^{-1}}{2 U}
$$

where $U(\phi) \equiv \frac{1}{2} \phi^{2}+V(\phi)$. It is easy to see that, if for a potential $U(\phi)$, Eq. (13) is satisfied with $R$ and $A$ such that $R / A^{2}=s(s+1), s$ being an integer, then the $\mathcal{A}(2 \rightarrow n)$ amplitudes at threshold vanish for any $n$ except for those given by Eq.(12). The inverse is also true, in the following sense: if $\mathcal{A}(2 \rightarrow n)$ amplitudes satisfy Eq.(12), then a potential $U(\phi)$ can be constructed, using Eqs.(8) and (9), which satisfies Eq.(13).

The above result enables us to find potentials with the nullification property. As a first, rather trivial, application, we examine the case of a monomial interaction, $U(\phi)=\frac{1}{2} \phi^{2}+\frac{1}{m !} \phi^{m}$. We find $R=m(m-1) / 2$ and $A=(m-2) / 2$, which gives $s=\frac{m}{m-2}$. This is an integer only for $m=3,4$. For $m=3, s=3$ and the amplitudes $\mathcal{A}(2 \rightarrow n)$ are non-zero only for $n=1,2,3$. For $m=4, s=2$ and the amplitudes vanish except for $n=2,4$. The case with $\phi^{3}$ as well as $\phi^{4}$ interactions is less trivial. The potential can be written generally as $U(\phi)=\frac{1}{2} \phi^{2}+\frac{\mu}{6} \phi^{3}+\frac{1}{24} \phi^{4}$. The application 
of Eq.(13) results in the determination of the constant $\mu: \mu= \pm \sqrt{3}$. This corresponds to the broken-symmetry case [3]. The latter is the only combination of $\phi^{3}$ and $\phi^{4}$ which leads to zeros of $\mathcal{A}(2 \rightarrow n)$ amplitudes. In this case $R=\frac{3}{2}$ and $A=\frac{1}{2}$, so $s=2$ and the non-zero amplitudes $\mathcal{A}(2 \rightarrow n)$ occur for $n=1,2$.

Equation (13) enables us to go beyond these known examples. We find that the sine-Gordon potential,

$$
U(\phi)=(1-\cos \phi)
$$

satisfies Eq.(13) with $A=1$ and $s=1$, so the only non-zero amplitude is $\mathcal{A}(2 \rightarrow 2)$. This is a well known property in $d=2$ space-time dimensions, where the theory is integrable and the nullification of $\mathcal{A}(2 \rightarrow n)$, for $n \geq 4$, holds for any kinematical configuration [15], due to the existence of an infinite set of conservation laws. We find that the nullification survives in all space-time dimensions at the kinemtaical threshold. Furthermore the same nullification occurs for the sinh-Gordon potential,

$$
U(\phi)=(\cosh \phi-1) .
$$

This can be verified directly, by solving the equation for $f(x)$ [3], which in the above cases is $f(x)=4 \tan ^{-1}\left(\frac{x}{4}\right)\left(=4 \tanh ^{-1}\left(\frac{x}{4}\right)\right)$ and then verify that Eq.(16) is of the form given by Eq.(10). It is also possible to construct 'customized' potentials in which $\mathcal{A}(2 \rightarrow n)$ is nonzero at threshold for only one value of $n$, and vanishes for all other values, by choosing appropriate values for $A$ and $s$ [16]. Obviously, these potentials satisfy Eq.(13).

In the case where we have in the Lagrangian the $\phi$ field coupled to other boson or fermion fields, the nullification of $\mathcal{A}(\chi \chi \rightarrow n \phi)$ or $\mathcal{A}(f \bar{f} \rightarrow n \phi)$ has exactly the same explanation in terms of reflectionless potentials [9]. The only difference is that in these cases the nullification occurs when the couplings and/or the masses of the particles obey definite relations among them. For instance, taking the coupling $\mathcal{L}_{i n t}=\frac{1}{4} \chi^{2} \phi^{2}+\frac{1}{24} \lambda_{4} \phi^{4}$ we find that for $g / \lambda_{4}=\frac{1}{6} s(s+1), \mathcal{A}(\chi \chi \rightarrow n \phi)$ vanish for $n \geq 2(s+1)$. This suggests that the search for a generalization of Eq.(13) in the case of several fields is undoubtedly interesting.

The nullification we study here does not exhaust all the possible cases where $\mathcal{A}(2 \rightarrow n)$ amplitudes vanish at threshold. For instance the unitarity-respecting toymodel potential described in [6] does not satisfy Eq.(13). Indeed, although we have found vanishing $\mathcal{A}(2 \rightarrow n)$ amplitudes for all $n$ even and larger than 4 , there is no nullification of the type discussed here, since the amplitudes are non-vanishing for all odd values of $n$.

It is worthwhile to note that Eq.(10) is the same as the equation describing the stability of $1+1$ dimensional solitons and is connected to scattering in the presence of solitonic backgrounds. It seems therefore that the nullification of $\mathcal{A}(2 \rightarrow n)$ amplitudes at threshold is a genuine dynamical effect, suggesting that for certain theories, some integrability properties emerge in the threshold kinematical configuration. The study of the (as yet ill-understood) relation between nullification at kinematical threshold and integrability in $d=2$ space-time dimensions could provide us with a new insight into multiboson production processes. 


\section{References}

[1] H. Goldberg, Phys.Lett. B246(1990),445.

J.M. Cornwall and G. Tiktopoulos, Phys.Rev.D46(1992),2105.

[2] M.B. Voloshin, 'Multiparticle amplitudes at zero energy and momentum in scalar theory', Univ. of Minnesota preprint TPI-MINN-92/1-T.

[3] E.N. Argyres, R. Kleiss and C.G. Papadopoulos, 'Amplitude estimates for multi-Higgs production at high energies', CERN-TH.6496/92

[4] M.B. Voloshin, Phys.Lett.B293(1992),389.

[5] E.N. Argyres, R. Kleiss and C.G. Papadopoulos, 'Cross-section estimates for multi-Higgs production at high energies', CERN-TH.6559/92.

[6] E.N. Argyres, R. Kleiss and C.G. Papadopoulos, 'Perturbative unitarity constraints on scalar self-interactions', CERN-TH.6629/92.

[7] M.B. Voloshin, 'Some properties of amplitudes at multi boson thresholds in spontaneously broken scalar theory', Univ. of Minnesota preprint TPI-MINN92/61-T.

[8] M.B. Voloshin, 'Summing one-loop graphs at multiparticle threshold', Univ. of Minnesota preprints TPI-MINN-92/45-T and TPI-MINN-92/46-T.

[9] M.B. Voloshin, 'Zeros of tree-level amplitudes at multiboson thresholds', Univ. of Minnesota preprint TPI-MINN-92/56-T.

[10] E.N. Argyres, R. Kleiss and C.G. Papadopoulos, 'Multiscalar production amplitudes beyond threshold', CERN-TH.6629/92.

[11] B.H. Smith, 'Summing one-loop graphs in a theory with broken symmetry', Univ. of Minnesota preprint TPI-MINN-92/50-T.

[12] L.S. Brown and C. Zhai, ' $2 \rightarrow n$ threshold production at tree level', Univ. of Washington preprint UW/PT-92-23.

[13] L.D. Landau and E.M. Lifshitz, Quantum Mechanics, non-relativistic theory, second edition (revised), Pergammon Press, §23, §25.

[14] L.S. Brown, Phys.Rev. D46(1992),R4125.

[15] A.B. Zamolodchikov and A.B. Zamolodchikov, Ann.Phys.120(1979),253.

[16] E.N. Argyres, R. Kleiss and C.G. Papadopoulos, in preparation. 